Autoren, die eine "Zuschrif" veroffentlichen wollen, soliten vor der Abfassung ihres Manuskripts unbedingt die „Hinweise for Autoren* lesen, die jeweils im Januarhef eines Jahrgangs nach dem Inhaltoverzeichnis gedruckt sind; auf Anforderung konnen sie auch von der Redaktion erhalten werden.

\section{1,4-Addition von Dihalogencarbenen an 1,3-Diene *}

Von Herbert Mayr" und Ulrich W. Heigl Professor Rolf Huisgen zum 65. Geburtstag gewidmet

Singulett-Carbene reagieren mit 1,3-Dienen oblicherweise unter 1,2-Addition zu Vinylcyclopropanen "I'. Theoretische Studien zeigen, daB der Ubergangszustand der

(') Prof. Dr. H. Mayr, Dipl.Chem. U. W. Heigl Institut för Chemie der Medizinischen Universitu Ratzeburger Allee 160, D-2400 Labeck

["9] Diese Arbeit wurde vom Fonds der Chemischen Industrie unterstetzt. Dr. I. Lojper. Erlangen, danken wir for eine Gasphasenthermolyse. ebenfalls Orbitalsymmetrie-erlaubten konzertierten 1,4Addition durch closed-shell-Repulsionen ${ }^{(22)}$, insbesondere die Vier-Elektronen-Wechselwirkung zwischen $\Psi_{1}($ Dien) und $\sigma$ (Carben), destabilisiert wird ${ }^{11.21}$. Nur in Ausnahmefallen, wie bei den Homo-1,4-Additionen von Dihalogencarbenen an Norbornadien [3a] oder der Benzvalen-Synthese durch intramolekulare Carben-Addition ${ }^{\text {DSt }}$, ist dieser Reaktionstyp bevorzugt. AuBer einigen Verbindungen, die als Folgeprodukte von 1,4-Addukten interpretiert wurden ${ }^{|\Delta|}$, sind 0.6\% 1,4-Addukt aus Dichlorcarben und 1,2Bis(methylen)cycloheptan der bisher einzige Hinweis auf eine intermolekulare 1,4-Addition eines Singulett-Carbens an ein 1,3-Dien ${ }^{[46]}$.

1,1,2,2,3,3-Hexamethyl-4,5-bis(methylen)cyclopentan 1 , das durch Bromierung und zweifache $\mathrm{HBr}$-Abspaltung aus Octamethylcyclopenten ${ }^{(S)}$ leicht hergestellt werden kann, ist ein für 1,4-Additionen pradestiniertes Modell, da es ein cis-fixiertes 1,3-Dien-System enthalt, bei dem der Zugang zu den Positionen 2 und 3 sterisch behindert ist.

Behandelt man 1 in Petrolether bei $0^{\circ} \mathrm{C} 4 \mathrm{~h}$ mit Chloroform und Kalium-tert-butylalkoholat, erhalt man $76 \%$ eines Gemisches aus 2a und 3a (73:27) (Tabelle 1). 2a ist unter den Reaktionsbedingungen stabil, da nach $30 \mathrm{~h}$ Reaktionszeit das gleiche Produktverhaltnis beobachtet wird. 
Analog entstehen mit Bromoform die Addukte 2b und 3b im Verhăltnis $70: 30$ in $80 \%$ Gesamtausbeute. 2a und 3a lassen sich chromatographisch trennen und durch ihre NMR-Spektren eindeutig charakterisieren (Tabelle 2)(6). Reines 3a erhălt man auch durch Blitzthermolyse des 2a/ 3a-Gemisches in einem Quarzrohr bei $350^{\circ} \mathrm{C}$.

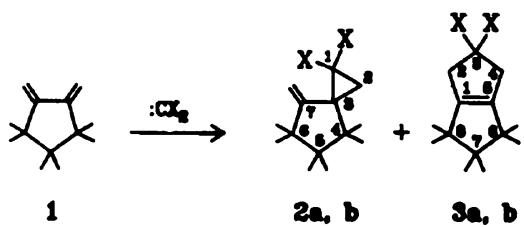

Tabelle 1. 1,2- und 1,4Addition von CX, an das 1,3-Dien 1

\begin{tabular}{|c|c|c|}
\hline$x$ & Reaktionsbedingungea & $2: 3$ \\
\hline - C & $\begin{array}{l}\mathrm{KO} B \mathrm{Bu} / \mathrm{CHCl}_{2} / \text { Petrolether } / 0^{\circ} \mathrm{C} \\
\mathrm{PhH}_{8} \mathrm{CCl}_{3} / \mathrm{Toluol} / 100^{\circ} \mathrm{C} / 1 \mathrm{~h}\end{array}$ & $\begin{array}{l}73: 27 \\
79: 21\end{array}$ \\
\hline b $\quad$ E & $\begin{array}{l}\mathrm{KOBBu} / \mathrm{CHBr} / \text { Petrolether } / 0^{\circ} \mathrm{C} \\
\mathrm{PhH} \mathrm{H}_{\mathrm{g}} \mathrm{CBr} / \mathrm{T}_{3} / \text { olvol } / 80^{\circ} \mathrm{C} / 1 \mathrm{~b}\end{array}$ & $\begin{array}{l}70: 30 \\
73: 27\end{array}$ \\
\hline
\end{tabular}

Erzeugt man die beiden Carbene aus Phenyl(trihalogenmethyl)quecksilber ${ }^{\prime \prime}$ in Toluol bei $100^{\circ} \mathrm{C}\left(\mathrm{CCl}_{2}\right)$ bzw. $80^{\circ} \mathrm{C}\left(\mathrm{CBr}_{2}\right)$, beobachtet man nach $1 \mathrm{~h}$ Reaktionszeit ein 2 :3-Verhaltnis von $79: 21$ bzw. 73 :27. Stündliche Probennahme zeigt, daB sich 2a und $2 b$ unter diesen Bedingungen langsam zu einem bisher nicht identifizierten Produkt zersetzen, so daß innerhalb von $5 \mathrm{~h}$ das $2: 3$-Verhaltnis auf $72: 28$ bzw. $65: 35$ abnimmt. Die langsame Verănderung des Produktverhăltnisses beweist jedoch eindeutig, $\mathrm{da} B$ die nach einer Stunde beobachteten Addukte 3a,b nicht als Umlagerungsprodukte von $2 \mathrm{a}$ bzw. $2 b$ gedeutet werden können.

Tabelle 2. '3-N-NMR-Daten der Carben-Additionopunkte 2 und 3. Bei Werten in Klammern ist die Zuordnung unklar.

\begin{tabular}{|c|c|c|c|c|c|c|c|c|}
\hline & C.1 & C.2 & C.3 & C.4 & C.S & $C-6$ & C-7 & $\begin{array}{l}-\mathrm{CH}_{2} \\
\text { oder } \mathrm{C}-8\end{array}$ \\
\hline 29 & $\begin{array}{l}67.63 \\
\text { Methylsi }\end{array}$ & $\begin{array}{c}26.29 \\
\text { ignale }\end{array}$ & $\begin{array}{c}(45.46) \\
\text { bei } 20.32\end{array}$ & $\begin{array}{c}\text { (46.31) } \\
\text { (doppelte }\end{array}$ & $\begin{array}{l}(47.81) \\
\operatorname{Int}), 23\end{array}$ & $\begin{array}{l}(48.95) \\
.07,26.94\end{array}$ & $\begin{array}{l}159.39 \\
28.64,2\end{array}$ & $\begin{array}{l}107.31 \\
0.66\end{array}$ \\
\hline $2 b$ & $\begin{array}{l}38.18 \\
\text { Methylsi }\end{array}$ & $\begin{array}{c}27.85 \\
\text { ignale }\end{array}$ & $\begin{array}{c}(45.39) \\
\text { bei 20.38, }\end{array}$ & $\begin{array}{c}(46.37) \\
20.41,23\end{array}$ & $\begin{array}{l}(46.68) \\
.49,27.9\end{array}$ & $\begin{array}{c}(49.58) \\
0,28.51,2\end{array}$ & $\begin{array}{l}160.37 \\
9.85\end{array}$ & 107.98 \\
\hline $3 a$ & $\begin{array}{l}145.00 \\
\text { Methylsi }\end{array}$ & $\begin{array}{r}51.39 \\
\text { ignale }\end{array}$ & $\begin{array}{l}92.22 \\
\text { bei } 21.78\end{array}$ & $\begin{array}{l}51.39 \\
\text { und } 23.8\end{array}$ & $\begin{array}{l}145.00 \\
\text { (doppel }\end{array}$ & $\begin{array}{l}(46.35) \\
\text { Ite } \ln t .)\end{array}$ & $(51.02)$ & $\triangle \mathrm{C}-6$ \\
\hline 3b & $\begin{array}{l}145.97 \\
\text { Methylsi }\end{array}$ & $\begin{array}{r}54.12 \\
\text { ignale }\end{array}$ & $\begin{array}{l}65.83 \\
\text { bei } 21.84\end{array}$ & $\begin{array}{l}54.12 \\
\text { und } 23.8\end{array}$ & $\begin{array}{l}145.97 \\
\text { (doppel }\end{array}$ & $\begin{array}{l}\text { (46.41) } \\
\text { Ite Int.) }\end{array}$ & (51.33) & $\triangle C_{-6}$ \\
\hline
\end{tabular}

Da bei der Reaktion von $\mathrm{CCl}_{2}$ und $\mathrm{CBr}_{2}$ mit 1 die 1,2 und 1,4-Addition in vergleichbarem AusmaB nebeneinander ablaufen, handelt es sich bei diesem Dien um ein geeignetes Modell, um die theoretischen Voraussagen zur 1,4-Addition ${ }^{[2]}$ zu aberprafen und den Vorschlägen aber einen zweistufigen Ablauf bestimmter Carben-Additionen ${ }^{10}$ nachzugehen. Es liegt auf der Hand, daB 1 als 1,3-Dien mit normaler 1,4, aber stark verringerter 1,2-Reaktivitat auch als mechanistische Sonde for weitere Reaktionen nutzbar ist, an denen sich 1,3-Diene ablicherweise als $x_{2}^{2}$. Einheiten beteiligen.

Eingegangen am 4. Marz 1985 [Z 1201]

[I] R. A. Moss, M. Jones, Jr. in R. A. Moss, M. Jones, Jr. (Hreg): Reactive Inrermediates, $V$ d. 2, Wiley, New Yort 1981, Kap. 3.

[2] a) H. Fujimoto, R. Hoffmann, J. Phys. Chem. 78 (1974) 1167; b) W. w.
Schoeller, E. Yurtsever, J. Am. Chem. Soc. 100 (1978) 7548; W. W. Schoeller, N. Aktekin, J. Chem. Soc. Chem. Commun. 1982, 20; C) andere Interpretation: N. L. Bauld, D. Wirth, J. Comput. Chem. 2 (1981) I.

[3] a) C. W. Jefford, nT. Kabengele, J. Kovace, U. Burger, Heb. Cuim. Acte S7 (1974) 104; spikere Arbeiten siehe [1]; b) U. Burger, O. Gandillon, Tetrahedron Lett. 1979. 4281.

[4] a) H. Hart, J. W. Ragron, Tetrahedron Letr. 24 (1983) 4891; L. W. Jenneskens, F. J. J. de Kanter, L. A. M. Turkenbure, H. J. R. de Boer, W. H. de Wolf, F. Bickelhaupt, Tetrahedron 10 (1984) 4401; b) L. A. M. Tuskenburg, W. H. de Wolf, F. Bickelhaupt, Tetrahedron Lett. 23 (1982) 769; siehe auch L. W. Jenneakens, W. H. de Wolr, F. Bickelhauph, Angew. Chem. 97 (1985) 568; Angew. Chem. Int. Ed. Enol. 24 (1985) Nr. 7.

[5] H. Klein, H. Mayr, Angew. Chem. 93 (1981) 1069; Angew. Chem. In. Ed Engl. 20 (1981) 1027.

[6] 'H-NMR (CDC1): 2a: $\delta=0.86,0.90,1.00,1.06$ (doppelce Int), 1.15 (5s: $\mathrm{CH}_{3}$ ), 1.53 (s: $\mathrm{CH}_{2}$ ), 4.64, 5.04 (2s: Vinyl-H); 20: $\delta-0.85,0.91,1.06,1.10$ (doppelte Int), 1.17 (5s: $\mathrm{CH}_{3}$ ) 1.70 (s: $\mathrm{CH}_{2}$ ), 4.60, 5.08 (2s: Vinyl-H); $3 \mathrm{a}$ : $\delta=0.87\left(8 ; 2 \mathrm{CH}_{3}\right), 0.96\left(8 ; 4 \mathrm{CH}_{3}\right), 3.15\left(8 ; 2 \mathrm{CH}_{2}\right) ; 34: \delta=0.86\left(8 ; 2 \mathrm{CH}_{3}\right)$ $0.95\left(8 ; 4 \mathrm{CH}_{3}\right), 3.38\left(8 ; 2 \mathrm{CH}_{2}\right)$

[7] D. Seyferth, Acc. Chem. Res. 5 (1972) 65.

[8] E. V. Dehmlow, R. Kramer, Angew. Chem. 96 (1984) 700; Angew. Chem. Int. Ed. Engl. 23 (1984) 706; B. Giese, W.-B. Lee, C. Neumann, bid. 9 (1982) 320 bzw. 21 (1982) 310. 